\title{
Impact of backlash, manufacturing deviations and friction on the load sharing factor of planetary gear systems
}

\author{
Athanassios Mihailidis ${ }^{1, *}$, Anastasios Moisiadis ${ }^{2}$, and Andreas Psarros ${ }^{1}$ \\ ${ }^{1}$ Lab of Machine Elements and Machine Design, School of Mechanical Engineering, Faculty of Engineering, Aristotle \\ University of Thessaloniki, 54624 Thessaloniki, Greece \\ ${ }^{2}$ Department of Mechanical Engineering, School of Technological Applications, University of Applied Sciences of Cen- \\ tral Macedonia, Serres, Greece
}

\begin{abstract}
Techniques and software tools, which were recently introduced by the authors, allowed for efficient automatic generation of 3D gear flanks and selective meshing of the gears of a simple planetary gear system with backlash and manufacturing imperfections. Friction of the meshing gear flanks was neglected. First results were promising and showed that even in geometrically perfect planetary gear systems the torque distribution is not uniform. It was further verified that pitch errors have a strong impact on the load distribution and that a self-aligning sun gear significantly enhances the torque distribution among the planets. In the current study, the procedure mentioned above is enhanced in several aspects. First, the tooth friction is considered. The friction coefficient is assumed constant along the path of contact; however different values for the sun-planet and planet-ring gear mesh may be given to account for the different contact conditions. Second, deviations are generated between given limits in a stochastic way. This feature significantly reduces the time needed to setup a model. Third, the entire analysis procedure is further automated by extensively employing Python scripting, enabling the solution of successive snapshots in much shorter time. Besides the torque distribution among the planets, the mesh load factor $K_{\gamma}$ and the deformation of the teeth, the planet bearing load is also shown.
\end{abstract}

\section{Introduction}

Planetary gearboxes are widely used in many applications like robotics, automotive, aircraft engines and wind turbines. Since torque is transmitted by multiple gear pairs, planetary systems offer very high torque density. Therefore, load distribution is an important design parameter to consider. Even if gears and carrier had perfect geometry, the load would not distribute equally among the planets, because the meshing stiffness varies along the path of contact. Moreover, load distribution depends on manufacturing and assembly errors, deformation and members support. Reference [1] contains an extensive literature review.

The impact of the sun's floating capability on the load distribution has been already investigated by the authors [2]. It was shown, that even if geometry was free from deviations, a floating sun design favours the load distribution. By considering the pitch error of just one active flank of the sun, the resulting load distribution was even more non-uniform. However, when the sun was let free to self-centre, the planets were almost equally loaded.

An experimental analysis was made by Kim et al [3, 4] taking into account the effect of carrier pinhole posi- tion errors on a two-stage planetary gear set. A simulation, considering one floating planetary gear, confirmed the experimental results with an accuracy better than than $10 \%$ [5]. The impact of many parameters, such as input torque, number of planets, pin diameter and bearing clearance, on the load sharing was presented. It was shown that increasing the input torque, the load sharing is improved. By increasing the pins diameter, the stiffness of the pins increases, limiting thus the deformation of the pin and reducing the ability of the planets to selfcentre. The same influence had the bearing clearance. Higher clearance results in better load distribution. It was further confirmed that increasing the number of planets increases the sensitivity to pinhole errors [6]. However, in the above research the gears were assumed perfect.

Nejad et al [7] among others investigated numerically and experimentally the effects of a floating sun gear in a wind turbine's planetary gearbox using a multi-body approach. Geometrical imperfections, pin hole location errors, eccentricity, sun modifications (lead crowning) and floating members were considered introducing the proper excitation.

Multibody dynamics approach offers models considering the mass forces of the members but without consid-

*Corresponding author: amih@,auth.gr 
ering deformation. On the other hand, finite element analysis, offers accurate geometry modelling and calculation of the deformation, but does not consider for the mass forces, since the analysis is quasi-static.

The current study extents the above-mentioned work of the authors [2] by calculating the load distribution for many successive snapshots. The efficiency of the procedure was greatly enhanced by using extensively Python scripting allowing thus to model errors on all active gear flanks. Furthermore, an additional design variant, i.e. a floating ring-gear, has been included in the analysis. Finally, the friction on the mating gear flanks was taken into account by assuming a constant friction coefficient.

\section{Planetary gear train}

In the current analysis, a simple planetary gear system with helical gears is considered. It contains a ring gear, a carrier with three equally spaced planets and a sun. Three cases are studied. In the first case, the sun rotates around a fixed axis. This model corresponds to a sun gear manufactured on the input shaft, which is supported by two bearings. In the second case, the sun is free to position itself between the planet gears. This model corresponds to a sun gear driven by a double spline articulated coupling. Finally, in the third case, the sun and the ring gear are free to position themselves between and around the planets. This model corresponds to a design where double spline couplings are used to connect the sun and the ring gear. The carrier and the planets are supported in the two bearings. They are considered very stiff compared to the gears, so they are modelled as rigid. The gear data of the planetary system are shown in Table 1 . They are the same as in [2], in order to allow for direct comparisons.

Table 1. Gear data

\begin{tabular}{|l|c|c|c|}
\hline & Sun & Planets & Ring \\
\hline Number of teeth $z$ & 13 & 24 & -65 \\
\hline $\begin{array}{l}\text { Addendum modification } \\
\text { coefficient } x\end{array}$ & 0.8 & 0.757 & 0.1017 \\
\hline Tip diameter $d_{a}[\mathrm{~mm}]$ & 16.85 & 28.65 & 67.6 \\
\hline Module $m_{n}[\mathrm{~mm}]$ & \multicolumn{3}{|c|}{10} \\
\hline Helix angle $\beta[$ degrees] & \multicolumn{3}{|c|}{21} \\
\hline Centre distance $a[\mathrm{~mm}]$ & \multicolumn{3}{|c|}{0.25} \\
\hline Facewidth $b[\mathrm{~mm}]$ & \multicolumn{3}{|c|}{ DIN 867} \\
\hline Bottom clearance $c_{p}[\mathrm{~mm}]$ & \multicolumn{3}{|c|}{} \\
\hline Basic rack & \multicolumn{3}{|c|}{}
\end{tabular}

\section{FE model}

A meshing algorithm, like that employed in Computational Fluid Dynamics applications was used in the current study, because it provides a smooth transition from fine to coarse mesh. To obtain reasonable contact results, it's needed to avoid coming in contact parts with coarse mesh. As shown in Figure 1, two areas of interest are meshed with different parameters. The first one consists of the intersections of the active flanks and the plane of action of each meshing gear. The second area is defined by the fillet at the roots of each tooth flank. The surfaces, which define these areas of interest, are grouped in special sets called in ANSA terminology "PROPERTIES" [8]. In the model, they are defined by hexahedral refinement boxes ("BCBOX"). When a surface is inside these boxes, different, properly chosen mesh parameters and quality criteria are applied in order to obtain a finer mesh and more accurate results.

The boundary conditions are applied in the model by multi point constrains ("MPC", "RIGID" type). The type "RIGID" was chosen since the gearbox foundation is assumed stiff. Significant improvement in the computational performance was accomplished by including in the contact domain only portions of surfaces that are expected to come in contact during the analysis of the current snapshot. This was achieved by properly developing the Python scripts.

Nodes defining MPC's and elements defining contact pairs are gathered in ANSA set entities, called "SET's". In every snapshot, the entire mesh is regenerated and therefore the previously defined sets are outdated. Consequently, using of ANSA generic entity builder ("GEB's") combined with scripting are employed in order refresh the contents of the set.
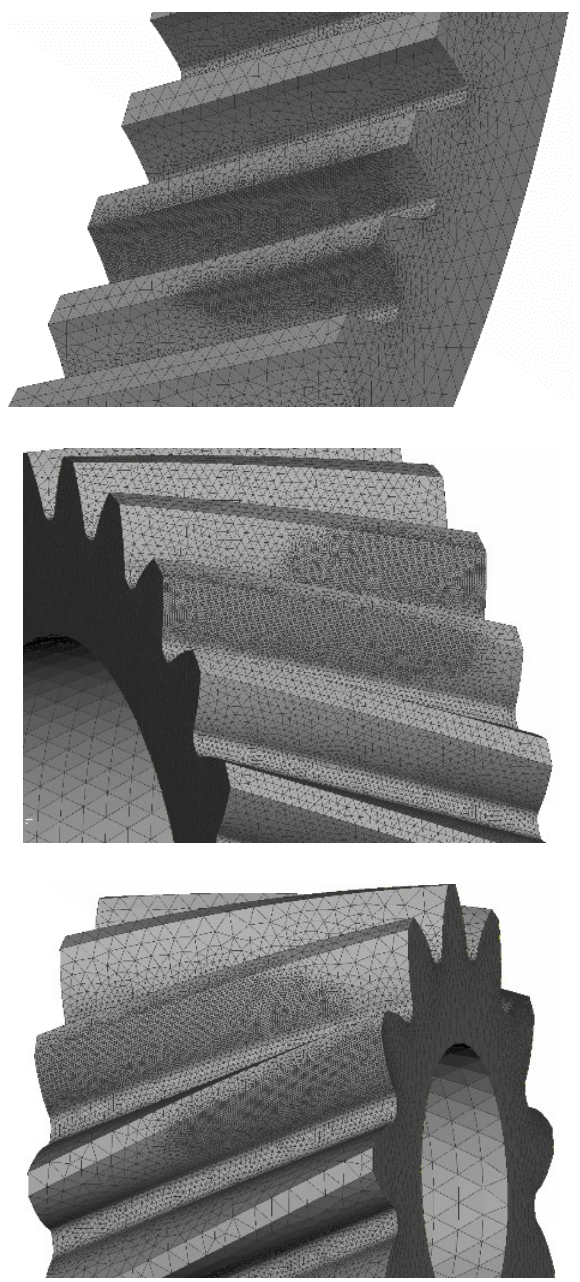

Fig 1. FE mesh of the ring, the planet and the sun gears.

In the root fillet area, the target element length was set to $0.15 \mathrm{~mm}$. The algorithm for these areas is named "SOLIDS STRUCTURAL MESH" and it generates a 
uniform mesh. Along the contact line the target element length was set to $0.08 \mathrm{~mm}$. In these areas the "CFD" algorithm was used. The growth rate parameter was chosen 2. The same algorithm was used in the remaining areas of the model. The growth rate was set 1.5. The allowable range of mesh size was set $0.08-1.5 \mathrm{~mm}$ in the entire model. Figure 1 shows the FE mesh of the gears.

The carrier was considered fixed. The input torque, $15 \mathrm{Nm}$, was applied to the sun gear. Torque output is from the ring gear. The friction coefficient was assumed 0.05 in all contacts.

Sun's angle of rotation between every snapshot was $4^{\circ}$. The described meshing procedure parameters resulted in models with about 1.5 million elements. Each snapshot required 30 minutes solving time on a computer equipped with 32 logical dual XEON CPU's at $2.7 \mathrm{GHz}$ and 128 GB RAM. The necessary data of each snapshot required almost $1.8 \mathrm{~GB}$ disk space. The procedure to create a snapshot was set-up once and then it was fully automated.

\section{Pitch error deviations}

The next step is to introduce manufacturing deviations in all active gear flanks of the model. Pitch error can be modelled by modifying the tooth thickness. The ANSA "MORPHING" tool is employed. Deviations are entered in all active gear flanks following the procedure described in detail in [2]. The deviations are generated automatically between given limits in a stochastic way following uniform distribution. The upper and lower limits are $[15 \mu \mathrm{m},-15 \mu \mathrm{m}]$. The magnitude of the deviations of each active flank is depicted in Figure 2. Depending on the torque direction, the active flanks of the sun and the ring gear are the ones on the right- or left-hand side. However, all flanks of the planet are active regardless the torque direction.

\section{Active flank error of sun}

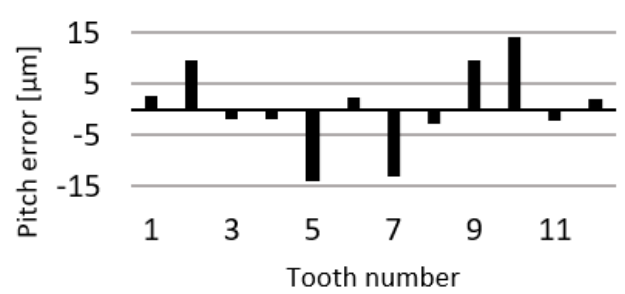

Active flank error of planet 1

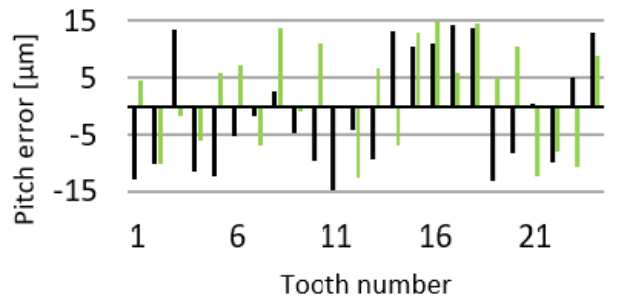

Active flank error of planet 2

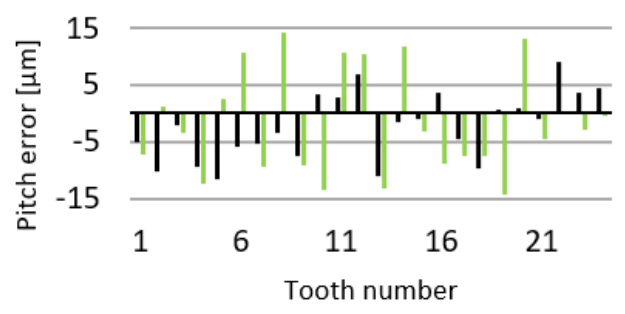

Active flank error of planet 3

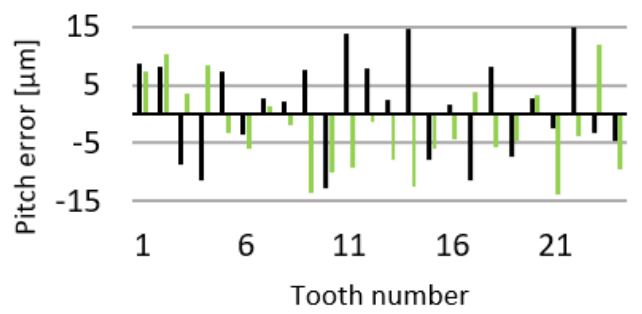

Active flank error of ring

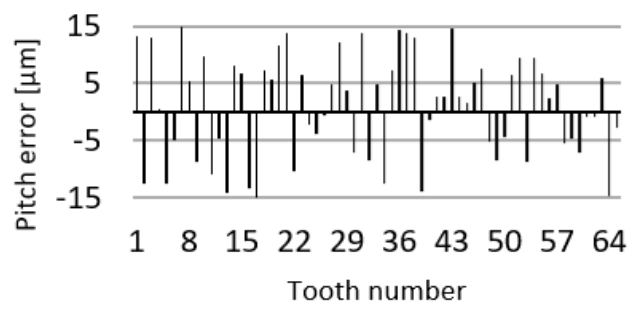

Fig 2. Pitch error deviations in active gear flanks.

\section{Solution scheme and results}

Figure 3 shows an overview of the procedure.

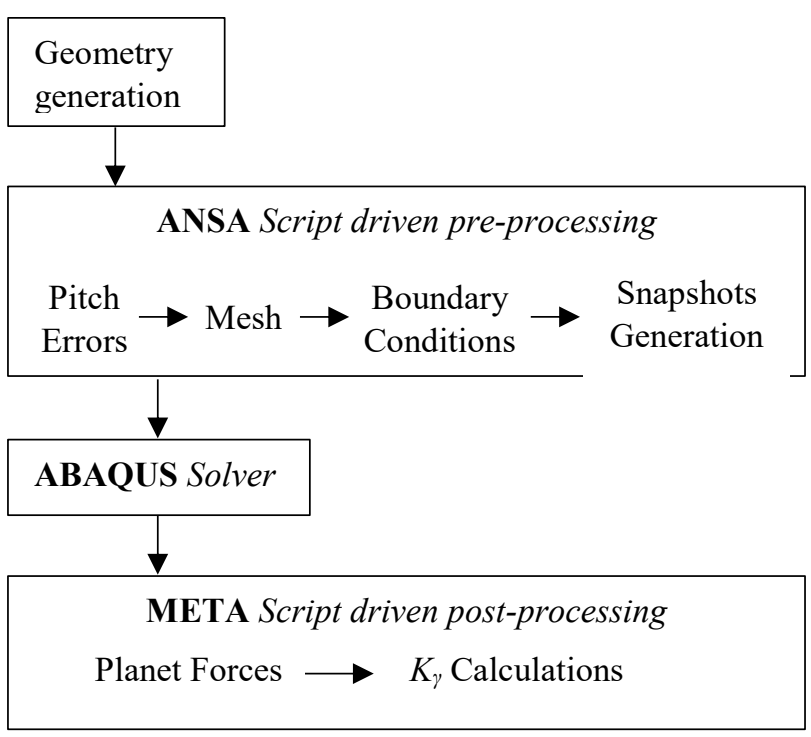

Fig 3. Overview of the workflow. 
The analysis is carried out using the ANSA pre-processor, ABAQUS solver and META post-processor. The following cases, shown in Table 2, were calculated:

- Case A: Geometrically perfect gears; sun gear allowed only to rotate around the axis of the planetary system.

- Case B: Geometrically perfect gears; sun gear allowed to centre itself between the planets. It can move as needed meaning that its axis may reach an off-centre and oblique position relative to the carrier axis. Only the movement in axial direction is restricted.

- Case C: Geometrically perfect gears; sun and ring gear allowed to centre themselves between the planets.

- Case D: All gears with pitch errors; sun gear allowed only to rotate as in case A.

- Case E: All gears with pitch errors; sun gear floating as in case B.

- Case F: All gears with pitch errors; sun and ring gears allowed to float as in case $\mathrm{C}$.

Table 2. Solved cases.

\begin{tabular}{|c|c|c|c|c|}
\hline & Sun & Planet & Ring & Deviations \\
\hline Case A & Fixed & Fixed & Fixed & No \\
\hline Case B & Free & Fixed & Fixed & No \\
\hline Case C & Free & Fixed & Free & No \\
\hline Case D & Fixed & Fixed & Fixed & Yes \\
\hline Case E & Free & Fixed & Fixed & Yes \\
\hline Case F & Free & Fixed & Free & Yes \\
\hline
\end{tabular}

\subsection{Load sharing factor}

The load sharing factor $K_{\gamma}$ is defined by the following equations.

$$
\begin{gathered}
K_{\gamma}=\frac{\max _{i=1}^{f}\left(T_{\imath}\right)}{T_{c} / f} \\
T_{i}=F_{i} a \\
T_{c}=\left|-T_{\text {in }}-T_{\text {out }}\right|=T_{\text {in }}\left|-1-\frac{z_{s}}{Z_{R}}\right|
\end{gathered}
$$

where:

$T_{i}$ : Torque acting on each planet's centre

$T_{c}$ : Supporting torque of the carrier

$F_{i}$ : Circumferential force at the centre of planet

$a$ : Centre distance of the planetary system

$f$ : Number of planets

$z_{S}$ : Number of teeth of the sun gear

$z_{R}$ : Number of teeth of the ring gear

Figure 4 shows the load sharing factor $K_{\gamma}$ for successive snapshots over the sun's rotation angle. At first, the favourable effect of floating members can be clearly seen, regardless the existence of pitch errors. In geometrically perfect gears, due to varying stiffness along the path of contact, the torque distribution is not uniform. In case A it seems to vary periodically during rotation. Second, flanks with stochastic pitch errors result in random torque distribution (Case D). In some snapshots one of the planets does not transmit any torque at all. Even in designs that allow only the sun gear to float (cases B and E) the torque is distributed almost evenly. In more advanced designs, where both sun and ring gear are allowed to selfcentre (Cases $\mathrm{C}$ and $\mathrm{F}$ ), the torque distribution is again very close to uniform. It is noteworthy, that the improvement in load sharing is mainly achieved by the floating sun.
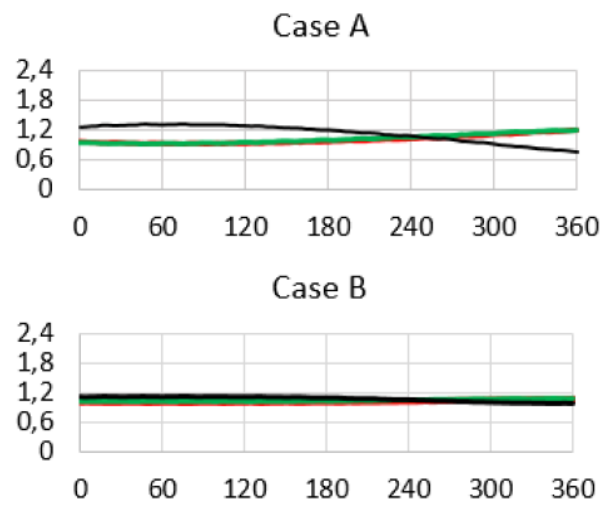

Case C
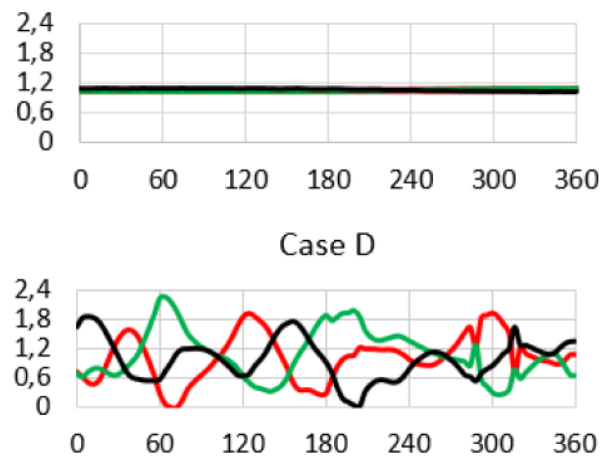

Case $\mathrm{E}$
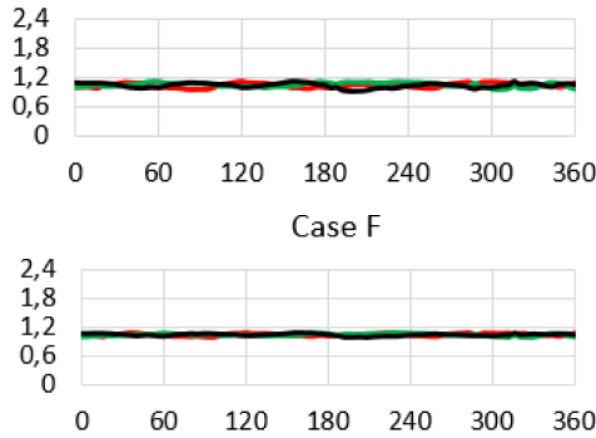

Fig 4. Load sharing factor over sun position as calculated for each case loading / design case.

Table 2 shows the calculated $K_{\gamma}$ values. The additional benefits of a floating ring gear are not significant.

Table 2. Calculated $K_{\gamma}$ for each loading / design case.

\begin{tabular}{|c|c|}
\hline & $K_{\gamma}$ \\
\hline Case A & 1.302 \\
\hline Case B & 1.124 \\
\hline Case C & 1.094 \\
\hline Case D & 2.264 \\
\hline Case E & 1.132 \\
\hline Case F & 1.092 \\
\hline
\end{tabular}


Previously published results [1] showed that the load sharing factor for planetary gear system with a fixed sun and deviations was about 2.2. Results agree quantitatively with current ones, even though the past model was 2D.

Considering friction obviously makes the model more realistic. Besides, it also has an important beneficial effect: It favours the convergence of the model, especially when considering floating members.

\subsection{Bearing forces}

Forces in each planet's bearing are calculated considering the forces acting on each planet's meshing (planet sun and planet - ring). The radial components of the bearing forces are calculated as reaction to the projection of the flank normal forces on the transverse plane. The axial force components, resulting from the flank normal forces in planet - sun and planet - ring gear have opposite directions. Thus, a tilting moment is generated that should be taken into account. Figures 5 and 6 present the loads (radial and axial) acting on the bearings of each planet. It should be noticed that radial force components are reduced in the floating member designs. However, axial force components are increased, although they are small compared to the radial ones.

Case A
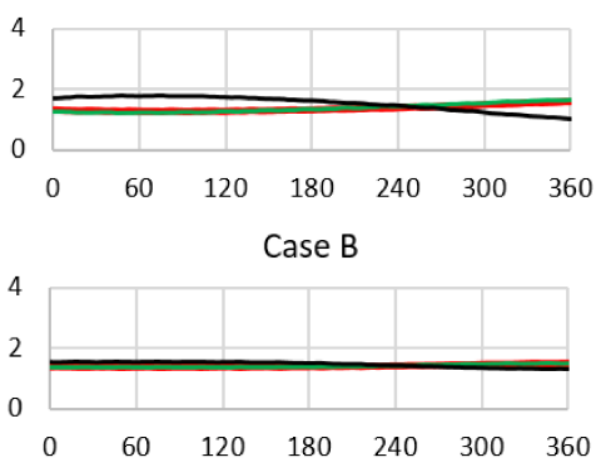

Case C

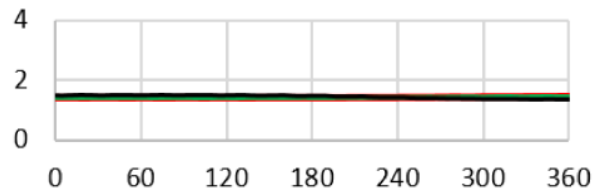

Case D

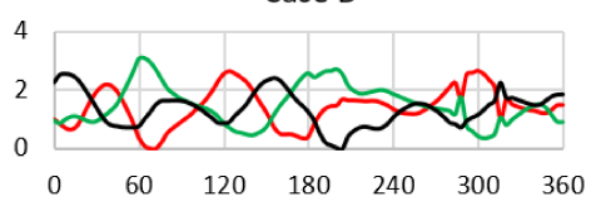

Case $\mathrm{E}$

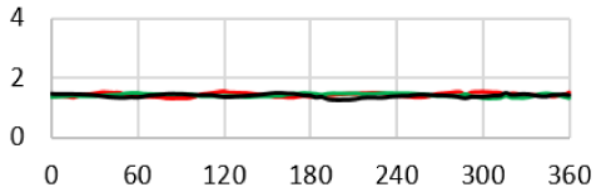

Case $\mathrm{F}$

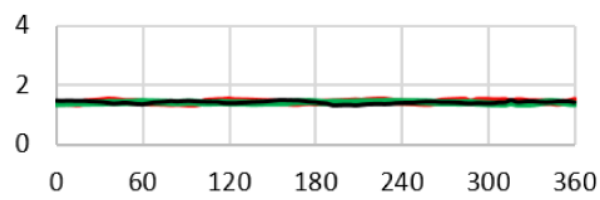

Fig 5. Transverse bearing force components in $\mathrm{kN}$ over sun position as calculated for in each loading / design case.

Case A
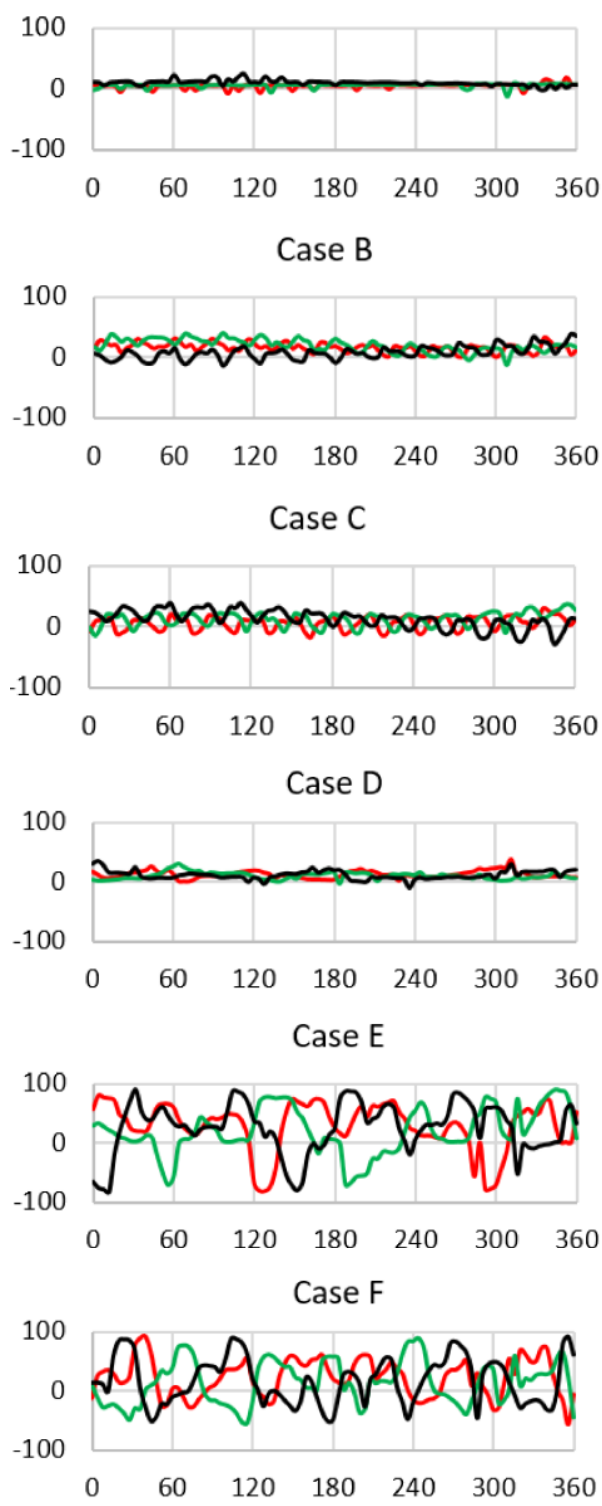

Fig 6. Axial bearing force component in $\mathrm{N}$ over sun position as calculated for in each loading / design case.

Figure 7 shows the deformation (magnitude, case F) of the members of the planetary gearbox. It should be noticed that the deformation of each planet is different due to errors imposed to the model. 

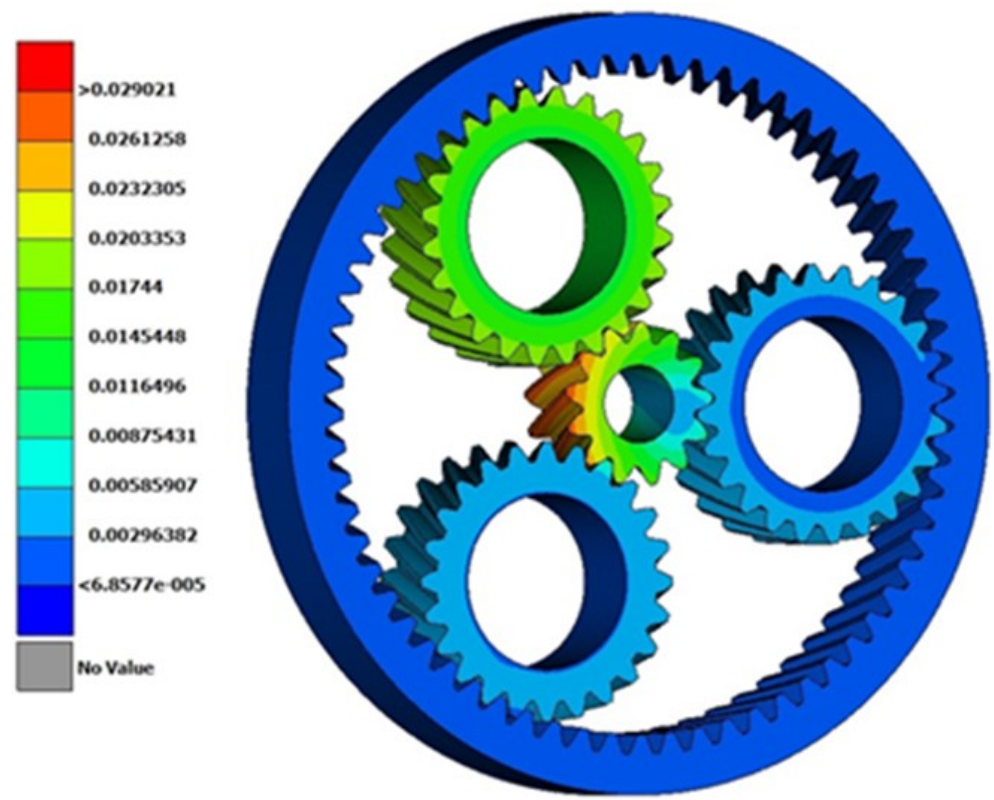

Fig 7. Displacements (magnitude) of all members of the gearbox (Case F, non-ideal gear flanks).

\section{Conclusions}

A multi snapshot 3D analysis of a simple planetary gear system consisting helical gears with pitch errors in all active gear flanks was made. Friction was imposed making the model more realistic but also much easier to solve, especially when simulating designs with floating members. The process was fully automated using Python scripting. Results show the beneficial effect of floating members in torque distribution. Even with the sun being the only floating member the load is distributed almost equally. Results concerning floating sun agreed with reference [6]. Even though the design of a planetary gearbox with floating members is more complex, it pays off since it results in a more uniform load sharing. Next steps of the research may include analysis of floating planets and other types of errors in flanks and in carrier. In addition, the stiffness of other members should be considered in order to make the model more accurate.

This project is supported by BETA CAE Systems, which provides the ANSA and $\mu$ ETA software.

\section{References}

1. A. Mihailidis, I. Panagiotidis, E. Bouras, E. Athanasopoulos: Finite element method based analysis of planetary gear systems considering backlash and manufacturing errors. International Conference on Gears 2015, 5-7 October, Munich, Germany, VDI Berichte 2255, 2015, pp. 79-88.

2. A. Mihailidis, G. Korbetis, N. Drivakos, I. Nerantzis, Finite element method based analysis of planetary gear systems considering backlash and manufacturing deviations, Power Transmission Engineering, June 2018, 48-52.

3. Jeong-Gil Kim, Young-Jun Park, Geun-Ho Lee, Jae-Hoon Kim: An experimental study on the effect of carrier pinhole position errors on planet gear load sharing. International Journal of Precision Engineering and Manufacturing, (2016), Vol 17, No 10, pp. 1305-1312.

4. Jeong-Gil Kim, Young-Jun Park, Geun-Ho Lee, Sang-Dae Lee, Joo-Young Oh: Experimental study on the carrier pinhole position error affecting dynamic load sharing of planetary gearboxes. International Journal of Precision Engineering and Manufacturing, (2018), Vol 19, No 6, pp. 881-887.

5. Jeong-Gil Kim, Young-Jun Park, Sang-Dae Lee, Joo-young Oh, Jae-Hoon Kim, Geun-Hoo Lee: Influence of the carrier pinhole position errors on the load sharing of a planetary gear train. International Journal of Precision Engineering and Manufacturing, (2018), Vol 19, No 4, pp. 537-543.

6. Singh A. Application of a System Level Model to Study the Planetary Load Sharing Behavior. ASME. J. Mech. Des. 2004;127(3):469-476.

7. Nejad, A. R., Xing, Y., Guo, Y., Keller, J., Gao, Z., and Moan, T. (2015) Effects of floating sun gear in a wind turbine's planetary gearbox with geometrical imperfections. Wind Energy, 18: 2105-2120.

8. BETA CAE Systems S.A. ANSA version 19.1.0 User's Guide, 2019.

9. BETA CAE Systems S.A. META version 19.1.0 User's Guide, 2019. 